\title{
Application of meshless EFG method in fluid flow problems
}

\author{
I V SINGH \\ Mechanical Engineering Group, Birla Institute of Technology and Science, \\ Pilani 333 031, India \\ e-mail: ivsingh@bits-pilani.ac.in
}

MS received 7 May 2003; revised 1 December 2003

\begin{abstract}
This paper deals with the solution of two-dimensional fluid flow problems using the meshless element-free Galerkin method. The unknown function of velocity $u(\mathbf{x})$ is approximated by moving least square approximants $u^{h}(\mathbf{x})$. These approximants are constructed by using a weight function, a monomial basis function and a set of non-constant coefficients. The variational method is used for the development of discrete equations. The Lagrange multiplier technique has been used to enforce the essential boundary conditions. A new exponential weight function has been proposed. The results are obtained for a two-dimensional model problem using different EFG weight functions and compared with the results of finite element and exact methods. The results obtained using proposed weight functions (exponential) are more promising as compared to those obtained using existing weight functions (quartic spline and Gaussian).
\end{abstract}

Keywords. Meshless method; element-free Galerkin method; steady state analysis; transient analysis; two-dimensional fluid flow.

\section{Introduction}

The finite element method (FEM) has been established as a very powerful numerical technique for the analysis of space domain problems having arbitrary shapes. It has been observed that in the finite element method, mesh generation is a far more time-consuming and expensive task than the assembly and solution of the finite element equations. Moreover, there are certain classes of problems for which FEM is difficult, or even impossible to apply. For such problems, it has become necessary to find the methods, which may be somewhat more expensive from the viewpoint of computer time but require less time in the preparation of data. Recently, a class of new methods, known as meshless methods, has been developed. Various meshless methods belonging to this family are: smooth particle hydrodynamics (Monaghan 1988, 1992), diffuse element method (Nayroles et al 1992), reproducing kernel particle method (Liu et al 1995), the method of finite spheres (De \& Bathe 2000), free mesh method (Yagawa \& Yamada 1996), local boundary integral equation (LBIE) method (Zhu et al 1998), the partition of unity

A list of symbols is given at the end of the paper 
method (Babuska \& Melenk 1997), element-free Galerkin (EFG) method (Belytschko et al 1994a, 1996; Lu et al 1994; Dolbow \& Belytschko 1998; Liu \& Gu 2000), meshless local Petrov-Galerkin (MLPG) method (Atluri \& Zhu 1998), natural element method (Sukumar et al 1998) and natural neighbour Galerkin method (Sukumar et al 2001).

Among all these meshless methods, the element-free Galerkin method (EFG) method has been successfully used to solve the problems of plates and shells (Krysl \& Belytschko 1996a, 1996b), fracture mechanics and wave propagation (Belytschko et al 1994b; Belytschko \& Tabbara 1996, Lu et al 1995; Sukumar et al 1997), NDT (Xuan et al 2001), electromagnetic field (Cingoski et al 1998) and heat transfer (Singh et al 2002, 2003a, 2003b).

In this paper, we have used the meshless element-free Galerkin (EFG) method for solving two-dimensional fluid flow problems and have proposed a new exponential weight function. In the EFG method, function over the solution domain requires only a set of nodes, and does not require element connectivity. Integration over the solution domain requires only a simple integration cell to obtain the solution. Variational technique has been used for the discretization of the governing equations for two-dimensional fluid flow problems. Lagrange multiplier method is used to enforce the essential boundary conditions. FORTRAN programs have been developed for two-dimensional transient and steady state analysis of fluid flow problems. The results are obtained for a model problem and compared with the results of exact (Appel et al 1959) and finite element (Reddy 1993) methods.

\section{The element-free Galerkin method}

The discretization of the governing equations by element-free Galerkin method requires moving least square interpolation functions which are made up of three components: a weight function associated with each node, a monomial basis function and a set of non-constant coefficients. The weight function is non-zero over a small neighbourhood at a particular node, called support of the node.

\subsection{Moving least square approximation}

Using moving least square approximation, the unknown function $u(y, z)$ is approximated by $u^{h}(y, z)$ over the two-dimensional domain $\Omega$ (Belytschko et al 1994a)

$$
u^{h}(y, z)=\sum_{j=1}^{m} p_{j}(y, z) a_{j}(y, z) \equiv \mathbf{p}^{T}(y, z) \mathbf{a}(y, z) \equiv \mathbf{p}^{T}(x) \mathbf{a}(\mathbf{x})
$$

where,

$$
\begin{aligned}
& m=\text { number of terms in the basis, } \\
& p_{j}(y, z)=\text { monomial basis function, } \\
& a_{j}(y, z)=\text { non-constant coefficients, } \\
& \mathbf{x}^{T}=\left[\begin{array}{ll}
y & z
\end{array}\right], \mathbf{p}^{T}(x)=\left[\begin{array}{lll}
1 & y & z
\end{array}\right] .
\end{aligned}
$$

The coefficients $a_{j}(\mathbf{x})$ are found by minimizing the quadratic functional $J(\mathbf{x})$ given by

$$
J(\mathbf{x})=\sum_{I=1}^{n} w\left(\mathbf{x}-\mathbf{x}_{I}\right)\left\{\sum_{j=1}^{m} p_{j}\left(\mathbf{x}_{I}\right) a_{j}(\mathbf{x})-u_{1}\right\}^{2},
$$


where $w\left(\mathbf{x}-\mathbf{x}_{I}\right)$ is a weight function which is non-zero over a small domain, called domain of influence. $n$ is the number of nodes in the domain of influence.

The minimization of $J(\mathbf{x})$ w.r.t. $\mathbf{a}(\mathbf{x})$ leads to the following set of equations

$$
\mathbf{a}(\mathbf{x})=\mathbf{A}^{-1}(\mathbf{x}) \mathbf{B}(\mathbf{x}) \mathbf{T},
$$

where $\mathbf{A}$ and $\mathbf{B}$ are given as

$$
\begin{aligned}
\mathbf{A} & =\sum_{I=1}^{n} w\left(\mathbf{x}-\mathbf{x}_{I}\right) \mathbf{p}\left(\mathbf{x}_{I}\right) \mathbf{p}^{T}\left(\mathbf{x}_{I}\right) \\
& =w\left(\mathbf{x}-\mathbf{x}_{1}\right)\left[\begin{array}{ccc}
1 & y_{1} & z_{1} \\
y_{1} & y_{1}^{2} & y_{1} z_{1} \\
z_{1} & y_{1} z_{1} & z_{1}^{2}
\end{array}\right]+\ldots w\left(\mathbf{x}-\mathbf{x}_{n}\right)\left[\begin{array}{ccc}
1 & y_{n} & z_{n} \\
y_{n} & y_{n}^{2} & y_{n} z_{n} \\
z_{n} & y_{n} z_{n} & z_{n}^{2}
\end{array}\right], \\
\mathbf{B}(\mathbf{x}) & =\left[w\left(\mathbf{x}-\mathbf{x}_{1}\right) \mathbf{p}\left(\mathbf{x}_{1}\right), \ldots w\left(\mathbf{x}-\mathbf{x}_{n}\right) \mathbf{p}\left(\mathbf{x}_{n}\right)\right] \\
& =\left[w\left(\mathbf{x}-\mathbf{x}_{1}\right)\left[\begin{array}{c}
1 \\
y_{1} \\
z_{1}
\end{array}\right], \ldots w\left(\mathbf{x}-\mathbf{x}_{n}\right)\left[\begin{array}{c}
1 \\
y_{n} \\
z_{n}
\end{array}\right]\right] \\
\mathbf{u}^{T} & =\left[u_{1}, u_{2}, \ldots u_{n}\right] .
\end{aligned}
$$

By substituting (3) in (1), the MLS approximants can be defined as

$$
u^{h}(\mathbf{x})=\sum_{I=1}^{n} \Phi_{1}(\mathbf{x}) u_{I}=\boldsymbol{\Phi}(\mathbf{x}) \mathbf{u}
$$

where the shape function $\Phi_{I}(\mathbf{x})$ is defined by

$$
\Phi_{I}(\mathbf{x})=\sum_{j=0}^{m} p_{j}(\mathbf{x})\left(\mathbf{A}^{-1}(\mathbf{x}) \mathbf{B}(\mathbf{x})\right)_{j I}=\mathbf{p}^{T} \mathbf{A}^{-1} \mathbf{B}_{I} .
$$

The derivative of the shape function is given as

$$
\Phi_{I, \mathbf{x}}(\mathbf{x})=\left(\mathbf{p}^{T} \mathbf{A}^{-1} \mathbf{B}_{I}\right)_{, \mathbf{x}}=\mathbf{p}_{, \mathbf{x}}^{T} \mathbf{A}^{-1} \mathbf{B}_{I}+\mathbf{p}^{T}\left(\mathbf{A}^{-1}\right)_{, \mathbf{x}} \mathbf{B}_{I}+\mathbf{p}^{T} \mathbf{A}^{-1}\left(\mathbf{B}_{I}\right)_{, \mathbf{x}} \cdot
$$

\subsection{Weight function description}

The weight function is non-zero over a small neighbourhood of $\mathbf{x}_{I}$, called the domain of influence of node $I$. The shape of this domain is arbitrary but in the present analysis rectangular domain is preferred (Belytschko et al 1994a). The choice of weight function $w\left(\mathbf{x}-\mathbf{x}_{I}\right)$ affects the resulting approximation $u^{h}\left(\mathbf{x}_{I}\right)$. In this paper, a new weight function namely exponential weight function has been proposed. The different weight functions used in the present analysis can be written as a function of normalized radius $r$ as the quartic-spline weight function (Belytschko et al 1996),

$$
w\left(\mathbf{x}-\mathbf{x}_{1}\right)=w(r)=\left\{\begin{array}{cc}
1-6 r^{2}+8 r^{3}-3 r^{4}, & 0 \leq r \leq 1 \\
0 & r>1
\end{array}\right\},
$$


Gaussian weight function (Belytschko et al 1995),

$$
w\left(\mathbf{x}-\mathbf{x}_{1}\right)=w(r)=\left\{\begin{array}{cc}
e^{-(2 \cdot 5 r)^{2}}, & 0 \leq r \leq 1 \\
0 & r>1
\end{array}\right\},
$$

the exponential weight function,

$$
w\left(\mathbf{x}-\mathbf{x}_{1}\right)=w(r)=\left\{\begin{array}{cc}
\frac{1}{100^{r}}, & 0 \leq r \leq 1 \\
0 & r>1
\end{array}\right\},
$$

where

$$
\begin{aligned}
& \left(r_{y}\right)_{I}=\frac{\left\|y-y_{I}\right\|}{d_{m y I}} \quad \text { and } \quad\left(r_{z}\right)_{I}=\frac{\left\|z-z_{I}\right\|}{d_{m z I}} \\
& d_{m y I}=d_{\max } c_{y I} \quad \text { and } \quad d_{m z I}=d_{\max } c_{z I}, d_{\max }=\text { scaling parameter, }
\end{aligned}
$$

$c_{y I}$ and $c_{z I}$ at node $I$, are the distances to the nearest neighbours. $d_{m y I}$ and $d_{m z I}$ are chosen such that the matrix is non-singular everywhere in the domain.

The weight function at any given point can be calculated as

$$
w\left(\mathbf{x}-\mathbf{x}_{I}\right)=w\left(r_{Y}\right) w\left(r_{z}\right)=w_{y} w_{z},
$$

where $w\left(r_{y}\right)$ or $w\left(r_{z}\right)$ can be calculated by replacing $r$ by $r_{y}$ or $r_{z}$ in the expression of $w(r)$.

The derivatives of the weight function are obtained as

$$
w_{, y}=\frac{\mathrm{d} w_{y}}{\mathrm{~d} y} w_{z}, w_{, z}=\frac{\mathrm{d} w_{z}}{\mathrm{~d} z} w_{y} .
$$

\section{Discretization of the governing equations}

The momentum equation for incompressible fluid flowing through the long and uniform duct is given as

$$
\mu\left(\frac{\partial^{2} u}{\partial y^{2}}+\frac{\partial^{2} u}{\partial z^{2}}\right)-\frac{d p}{d x}=\rho \frac{\partial u}{\partial t} .
$$

The Initial conditions are

$$
\text { at } t=0, \quad u=u_{\text {ini }}, \quad \text { in } \Omega .
$$

The essential boundary conditions are

at the surface $S_{1}, \quad u=u_{S_{1}}=0$,

at the surface $S_{2}, \quad u=u_{S_{2}}=0$,

at the surface $S_{3}, \quad u=u_{S_{3}}=0$,

at the surface $S_{4}, \quad u=u_{S_{4}}=0$.

The weak form of (12a) with the boundary conditions is obtained as

$$
-\int_{\Omega}\left[\mu\left(w_{, y} u_{, y}+w_{, z} u_{, z}\right)\right] \mathrm{d} \Omega+\int_{\Omega} w M \mathrm{~d} \Omega-\int_{\Omega} \rho w \dot{u} \mathrm{~d} \Omega=0 .
$$


The functional $\Pi(u)$ can be written as

$$
\Pi(u)=\int_{\Omega} \frac{\mu}{2}\left[u_{, y}^{2}+u_{, z}^{2}\right] \mathrm{d} \Omega+\int_{\Omega} \rho \dot{u} u \mathrm{~d} \Omega-\int_{\Omega} M u \mathrm{~d} \Omega .
$$

Enforcing essential boundary conditions using Lagrange multipliers, the functional $\Pi^{*}(u)$ is obtained as

$$
\begin{aligned}
\Pi^{*}(u)= & \int_{\Omega} \frac{\mu}{2}\left[u_{, y}^{2}+u_{, z}^{2}\right] \mathrm{d} \Omega+\int_{\Omega} \rho \dot{u} u \mathrm{~d} \Omega-\int_{\Omega} u M \mathrm{~d} \Omega+\int_{\Gamma_{1}} \lambda\left(u-u_{S_{1}}\right) \mathrm{d} \Gamma \\
& +\int_{\Gamma_{2}} \lambda\left(u-u_{S_{2}}\right) \mathrm{d} \Gamma+\int_{\Gamma_{3}} \lambda\left(u-u_{S_{3}}\right) \mathrm{d} \Gamma+\int_{\Gamma_{4}} \lambda\left(u-u_{S_{4}}\right) \mathrm{d} \Gamma
\end{aligned}
$$

Using the variational principle, (15) reduces to

$$
\begin{aligned}
\delta \Pi^{*}(u)= & \int_{\Omega} \mu\left[u_{, y}^{T} \delta u_{, y}+u_{, z}^{T} \delta u_{, z}\right] \mathrm{d} \Omega+\int_{\Omega} \rho \dot{u} \delta u \mathrm{~d} \Omega-\int_{\Omega} M \delta u \mathrm{~d} \Omega \\
& +\int_{\Gamma_{1}} \delta \lambda\left(u-u_{S_{1}}\right) \mathrm{d} \Gamma+\int_{\Gamma_{1}} \lambda \delta u \mathrm{~d} \Gamma+\int_{\Gamma_{2}} \delta \lambda\left(u-u_{S_{2}}\right) \mathrm{d} \Gamma+\int_{\Gamma_{2}} \lambda \delta u \mathrm{~d} \Gamma \\
& +\int_{\Gamma_{3}} \delta \lambda\left(u-u_{S_{3}}\right) \mathrm{d} \Gamma+\int_{\Gamma_{3}} \lambda \delta u \mathrm{~d} \Gamma+\int_{\Gamma_{4}} \delta \lambda\left(u-u_{S_{4}}\right) \mathrm{d} \Gamma+\int_{\Gamma_{4}} \lambda \delta u \mathrm{~d} \Gamma .
\end{aligned}
$$

Since $\delta u$ and $\delta \lambda$ are arbitrary in preceding equation, the following relations are obtained using (7),

$$
\begin{aligned}
{[\mathbf{K}]\{\mathbf{u}\}+[\mathbf{C}]\{\dot{\mathbf{u}}\}+[\mathbf{G}]\{\lambda\} } & =\{\mathbf{f}\}, \\
{\left[\mathbf{G}^{T}\right]\{\dot{\mathbf{u}}\} } & =\{\mathbf{q}\},
\end{aligned}
$$

where

$$
\begin{aligned}
K_{I J}= & \int_{\Omega}\left[\begin{array}{l}
\Phi_{I, y} \\
\Phi_{I, z}
\end{array}\right]^{T}\left[\begin{array}{ll}
\mu & 0 \\
0 & \mu
\end{array}\right]\left[\begin{array}{l}
\Phi_{J, y} \\
\Phi_{J, z}
\end{array}\right] \mathrm{d} \Omega, \\
C_{I J}= & \int_{\Omega} \Phi_{I}^{T}\left[\begin{array}{ll}
\rho & 0 \\
0 & \rho
\end{array}\right] \Phi_{J} \mathrm{~d} \Omega, \\
G_{I K}= & \int_{\Gamma_{1}} \Phi_{I} N_{K} \mathrm{~d} \Gamma_{1}+\int_{\Gamma_{2}} \Phi_{I} N_{K} \mathrm{~d} \Gamma_{2}+\int_{\Gamma_{3}} \Phi_{I} N_{K} \mathrm{~d} \Gamma_{3}+\int_{\Gamma_{4}} \Phi_{I} N_{K} \mathrm{~d} \Gamma_{4}, \\
f_{I}= & \int_{\Omega} M \Phi_{I} \mathrm{~d} \Omega, \\
q_{K}= & \int_{\Gamma_{1}} u_{S_{1}} N_{K} \mathrm{~d} \Gamma_{1}+\int_{\Gamma_{2}} u_{S_{2}} N_{K} \mathrm{~d} \Gamma_{2}+\int_{\Gamma_{3}} u_{S_{3}} N_{K} \mathrm{~d} \Gamma_{3}+\int_{\Gamma_{4}} u_{S_{4}} N_{K} \mathrm{~d} \Gamma_{4} .
\end{aligned}
$$




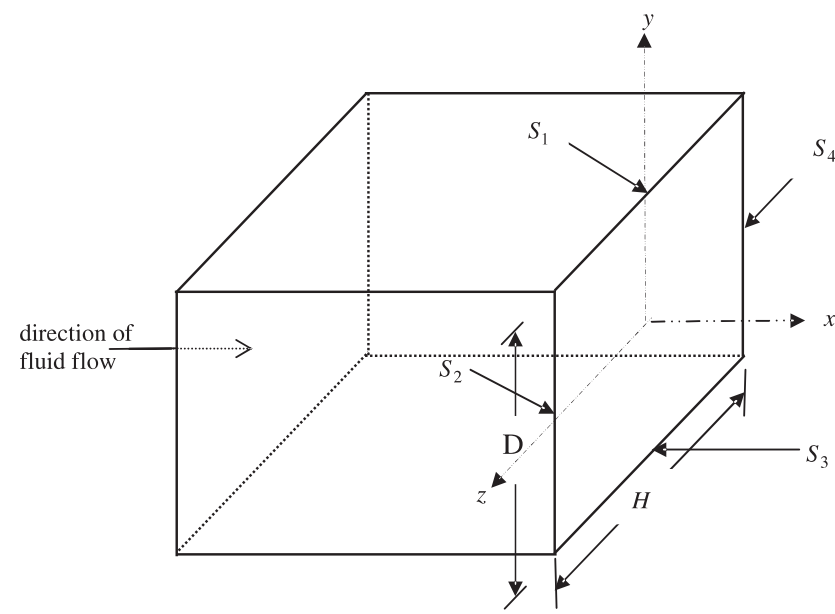

Figure 1. Cross-section of twodimensional model for fluid flow problem.

Using Crank-Nicolson method for time approximation, (17) can be written as

$$
\left[\begin{array}{l|l}
\mathbf{K}^{*}+\mathbf{C} & \mathbf{G} \\
\hline \mathbf{G}^{T} & 0
\end{array}\right]\left\{\begin{array}{l}
\mathbf{u}_{n^{\prime}} \\
\boldsymbol{\lambda}
\end{array}\right\}=\left\{\begin{array}{c}
\mathbf{R}_{n^{\prime}} \\
\mathbf{q}
\end{array}\right\},
$$

where

$$
\begin{aligned}
& \mathbf{R}_{n^{\prime}}=([\mathbf{C}]-(1-\alpha) \Delta t[\mathbf{K}])\{\mathbf{u}\}_{n^{\prime}-1}+\alpha \Delta t\{\mathbf{f}\}_{n^{\prime}}+(1-\alpha) \Delta t\{\mathbf{f}\}_{n^{\prime}-1}, \\
& \mathbf{K}^{*}=\alpha \Delta t[\mathbf{K}] .
\end{aligned}
$$

\section{Numerical results and discussion}

The parameters considered for the transient and steady analysis of the model shown in figure 1 are tabulated in table 1 . Table 2 shows a comparison of velocity obtained by EFG method for different weight functions with that obtained by finite element method at the location ( $y=D / 4, y=H / 4$ ) of the duct cross-section for 121 nodes. A similar comparison of

Table 1. Data for the 2-D transient and steady state fluid flow.

\begin{tabular}{ll}
\hline Parameters & Value of parameters \\
\hline Depth $(D)$ & $0 \cdot 10 \mathrm{~m}$ \\
Height $(H)$ & $0 \cdot 10 \mathrm{~m}$ \\
Pressure gradient $(-\partial P / \partial x)$ & $4000 \cdot 0\left(\mathrm{~N} / \mathrm{m}^{2} / \mathrm{m}\right)$ \\
Density of the fluid $(\rho)$ & $1000 \mathrm{~kg} / \mathrm{m}^{3}$ \\
Dynamic viscosity $(\mu)$ & $2 \cdot 5 \mathrm{Ns} / \mathrm{m}^{2}$ \\
All surface velocities $\left(u_{S}\right)$ & $0 \cdot 0 \mathrm{~m} / \mathrm{s}$ \\
Initial velocity $\left(u_{\text {ini }}\right)$ & $0 \cdot 0 \mathrm{~m} / \mathrm{s}$ \\
Time step $(\Delta t)$ & $0 \cdot 01 \mathrm{~s}$ \\
\hline
\end{tabular}


Table 2. Comparison of transient EFG results with FEM at the location $(y=D / 4, z=H / 4)$ of the duct cross-section.

\begin{tabular}{lcccc}
\hline & \multicolumn{4}{c}{ Velocity (m/s) } \\
\cline { 2 - 4 } & \multicolumn{4}{c}{ EFG } \\
\cline { 2 - 4 } Time(s) & Quartic spline & Gaussian & Exponential & FEM \\
\hline \multirow{2}{*}{0.00} & 0.0000 & 0.0000 & 0.0000 & 0.0000 \\
$0 \cdot 10$ & 0.3256 & 0.3235 & 0.3257 & 0.2994 \\
0.20 & 0.4895 & 0.4855 & 0.4896 & 0.4702 \\
0.30 & 0.5859 & 0.5805 & 0.5861 & 0.5711 \\
0.40 & 0.6442 & 0.6375 & 0.6444 & 0.6332 \\
0.50 & 0.6795 & 0.6719 & 0.6797 & 0.6718 \\
0.60 & 0.7009 & 0.6925 & 0.7012 & 0.6958 \\
0.70 & 0.7139 & 0.7050 & 0.7141 & 0.7107 \\
0.80 & 0.7217 & 0.7125 & 0.7220 & 0.7200 \\
0.90 & 0.7265 & 0.7170 & 0.7268 & 0.7258 \\
1.00 & 0.7294 & 0.7197 & 0.7297 & 0.7293 \\
\hline
\end{tabular}

velocity has also been shown in figure 2 at the centre $(y=0, z=0)$ of the duct cross-section for the same number of nodes.

The steady state analysis has been carried out for a model problem by using different EFG weight functions. Table 3 shows a comparison of the results obtained using EFG

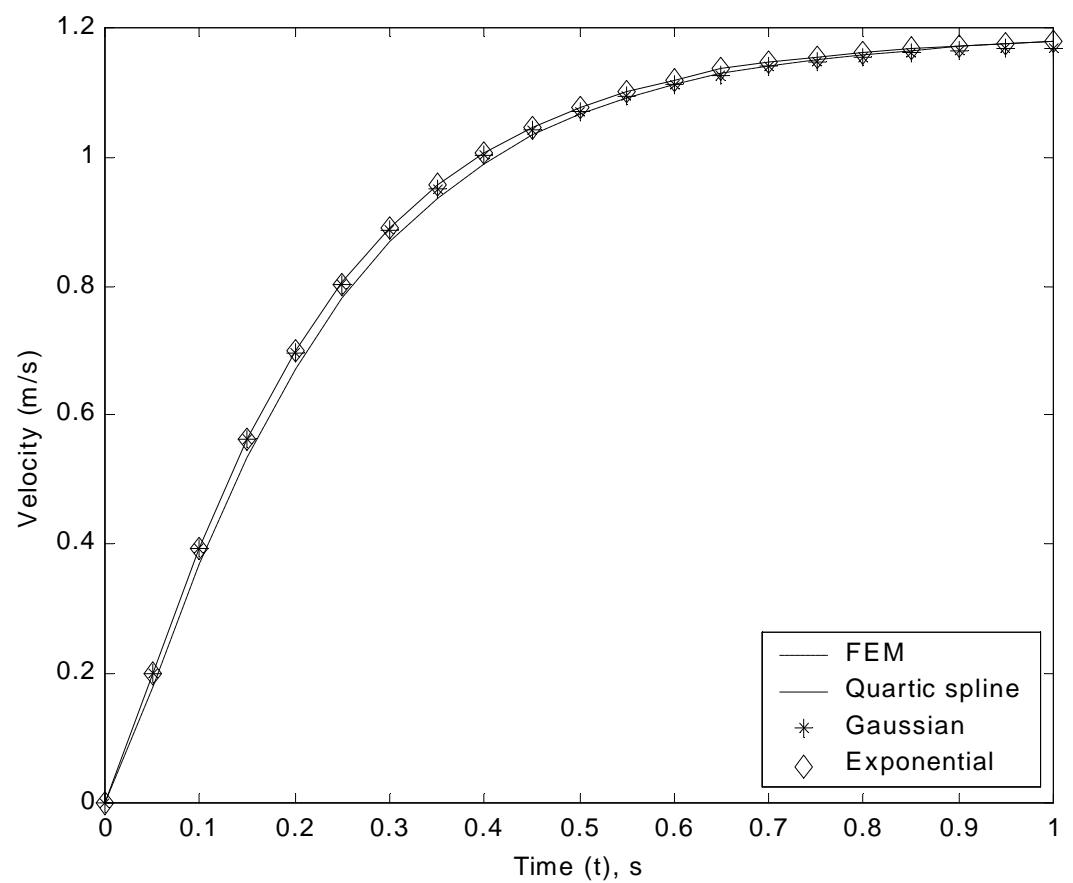

Figure 2. Comparison of transient EFG results with FEM at the centre $(y=0, z=0)$ of the duct cross-section. 
Table 3. Comparison of steady state EFG results for different weight functions with FEM and exact methods at the centre $(y=0, z=0)$ of the duct cross-section.

\begin{tabular}{|c|c|c|c|c|c|}
\hline \multirow{3}{*}{$\begin{array}{l}\text { No. of } \\
\text { nodes }\end{array}$} & \multicolumn{4}{|c|}{ Velocity (m/s) } & \multirow[b]{3}{*}{ Exact } \\
\hline & \multicolumn{3}{|c|}{ EFG } & \multirow[b]{2}{*}{ FEM } & \\
\hline & Quartic spline & Gaussian & Exponential & & \\
\hline 25 & 1.2338 & 1.1753 & 1.2358 & 0.9839 & $1 \cdot 1802$ \\
\hline 81 & 1.1916 & $1 \cdot 1776$ & $1 \cdot 1920$ & $1 \cdot 1619$ & $1 \cdot 1802$ \\
\hline 121 & 1.1869 & $1 \cdot 1780$ & $1 \cdot 1872$ & $1 \cdot 1696$ & 1.1802 \\
\hline 441 & 1.1808 & $1 \cdot 1786$ & $1 \cdot 1808$ & $1 \cdot 1764$ & $1 \cdot 1802$ \\
\hline
\end{tabular}

method with FEM and exact methods. The $L_{2}$-error has been calculated for different EFG weight functions and FEM with exact solution and is given in table 4 . The $L_{2}$-error has also been plotted in figure 3 for the calculation of rate of convergence. The rate of convergence for quarticspline, Gaussian, exponential and FEM are obtained as 1.0521, 0.9305, 1.0630 and 1.3709 respectively. From table 4 and figure 3, it is observed that the convergence of exponential weight function is better than those obtained using quartic spline and Gaussian weight functions. It can be also be noted that the accuracy in EFG results is more as compared with FEM results but the convergence of FEM results is better than EFG results.

Table 5 shows the effect of scaling parameter $\left(d_{\max }\right)$ on EFG results at the location $(y=$ $D / 4, z=H / 4$ ) of the duct cross-section. A similar comparison of results has also been shown in figure 4 at the centre $(y=0, z=0)$ of the duct cross-section. From these results, it is clear that the variation in EFG results with scaling parameter for exponential weight function is less as compared quartic spline and Gaussian weight functions. The scaling parameter $\left(d_{\max }\right)$ gives acceptable results for the range $1<d_{\max } \leq 1.5$.

It can be noted from the tables and figures that the EFG results are well converged and in good agreement with the results of finite element and exact methods. From the analysis, it is also clear that the proposed exponential weight function is more promising as compared to quatric spline and Gaussian weight functions. For higher accuracy, large number of nodes

Table 4. $L_{2}$-error in velocity for different weight functions of EFG and FEM.

\begin{tabular}{lrrrr}
\hline & \multicolumn{3}{c}{ EFG } & \\
\cline { 2 - 4 } $\begin{array}{l}\text { Elemental } \\
\text { area/cell } \\
\text { area }\left(\mathrm{m}^{2}\right)\end{array}$ & $\begin{array}{c}\text { Quartic spline } \\
\left(x 10^{-4}\right)\end{array}$ & $\begin{array}{c}\text { Gaussian } \\
\left(x 10^{-4}\right)\end{array}$ & $\begin{array}{c}\text { Exponential } \\
\left(x 10^{-4}\right)\end{array}$ & \multicolumn{1}{c}{$\begin{array}{c}\text { FEM } \\
\left(x 10^{-4}\right)\end{array}$} \\
\hline $0 \cdot 000625$ & 30.77780 & 32.20820 & 31.95243 & 214.93360 \\
0.000156 & 6.56262 & 2.21061 & 6.81253 & $39 \cdot 13477$ \\
$0 \cdot 000100$ & 3.82998 & $2 \cdot 07301$ & 3.97280 & 6.80464 \\
$0 \cdot 000025$ & 1.06004 & 1.76356 & 1.06089 & 2.88473 \\
\hline
\end{tabular}




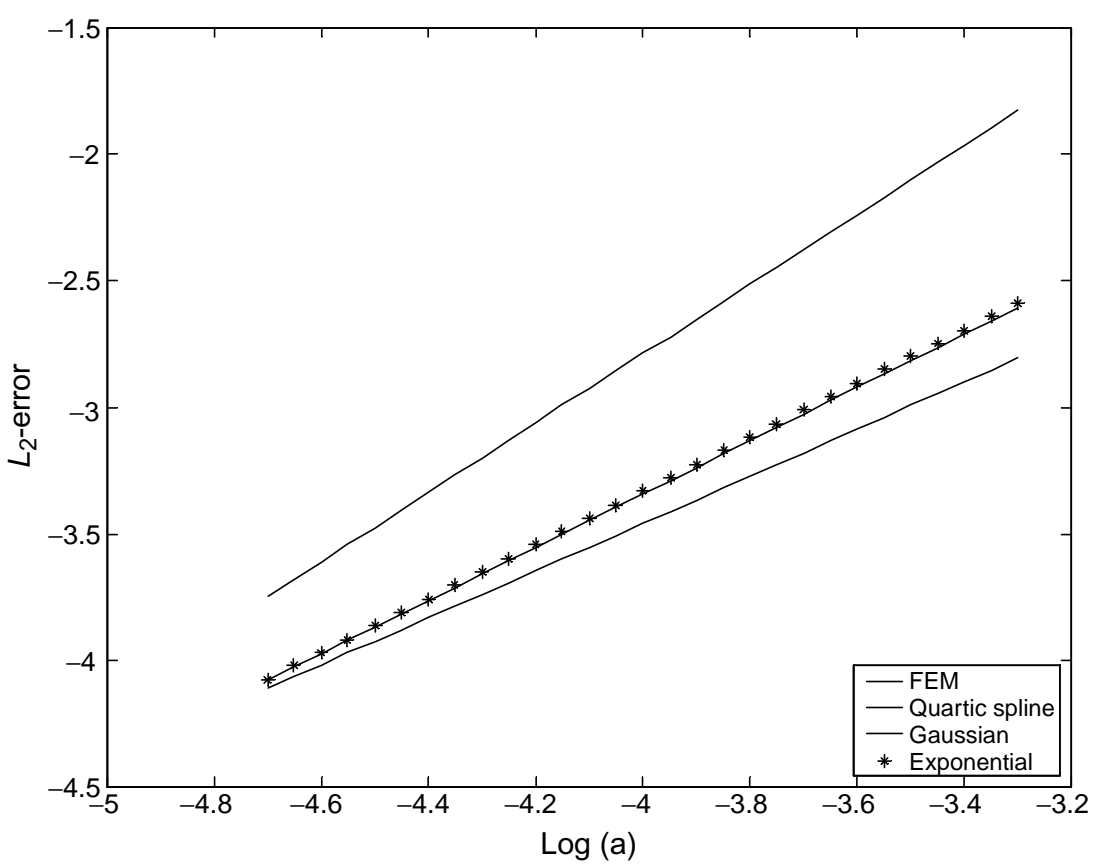

Figure 3. A plot of $L_{2}$-error for different weight functions of EFG and FEM.

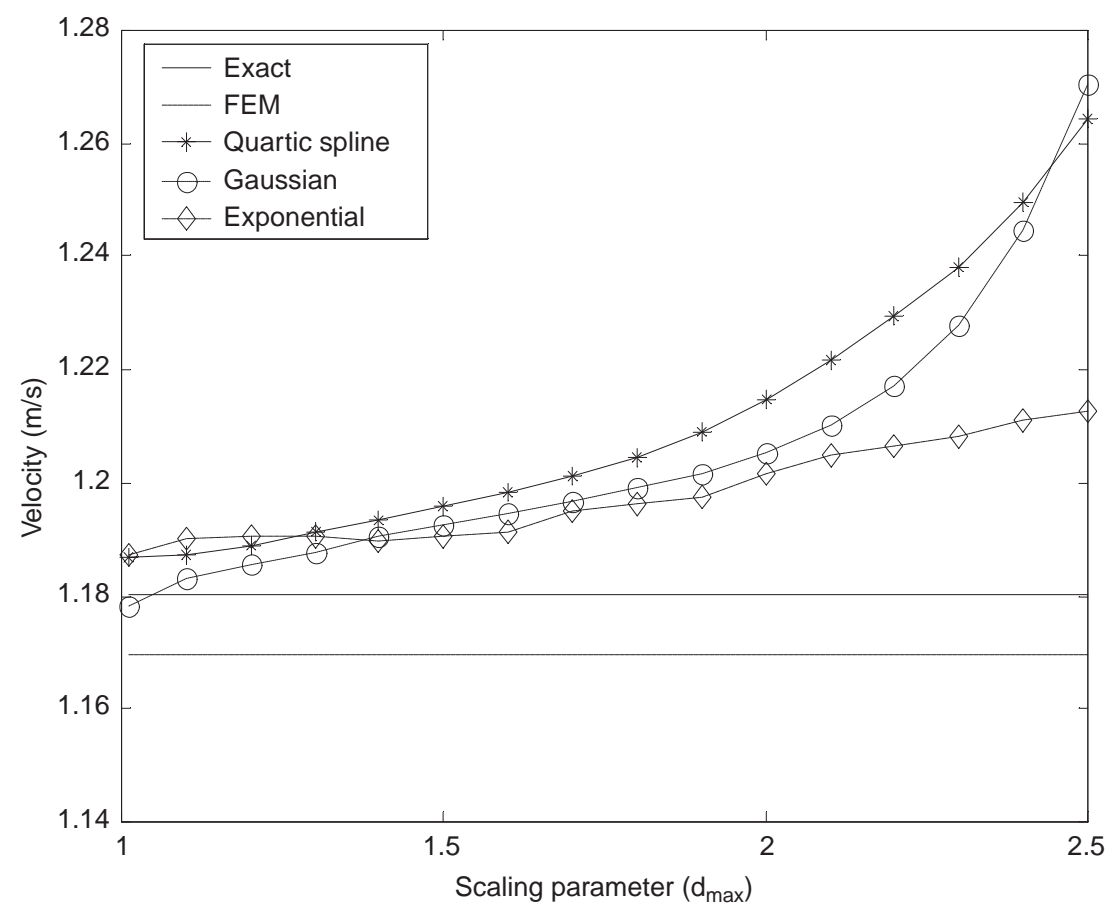

Figure 4. Effect of scaling parameter $\left(d_{\max }\right)$ on EFG results at the centre $(y=0, z=0)$ of the duct cross-section. 
Table 5. Effect of scaling parameter $\left(d_{\max }\right)$ on EFG results for different weight functions at the location $(y=D / 4, z=H / 4)$ of the duct cross-section.

\begin{tabular}{lccccc}
\hline & \multicolumn{5}{c}{ Velocity $(\mathrm{m} / \mathrm{s})$} \\
\cline { 2 - 4 } & \multicolumn{5}{c}{ EFG } \\
\cline { 2 - 4 } $\begin{array}{l}\text { Scaling } \\
\text { parameter }\end{array}$ & Quartic spline & Gaussian & Exponential & FEM & Exact \\
\cline { 2 - 5 } 1.01 & 0.7338 & 0.7299 & 0.7341 & 0.7139 & 0.7260 \\
1.1 & 0.7333 & 0.7334 & 0.7412 & 0.7139 & 0.7260 \\
1.2 & 0.7369 & 0.7368 & 0.7417 & 0.7139 & 0.7260 \\
1.3 & 0.7428 & 0.7395 & 0.7420 & 0.7139 & 0.7260 \\
1.4 & 0.7461 & 0.7432 & 0.7336 & 0.7139 & 0.7260 \\
1.5 & 0.7499 & 0.7457 & 0.7389 & 0.7139 & 0.7260 \\
1.6 & 0.7517 & 0.7480 & 0.7402 & 0.7139 & 0.7260 \\
1.7 & 0.7516 & 0.7502 & 0.7456 & 0.7139 & 0.7260 \\
1.8 & 0.7520 & 0.7512 & 0.7473 & 0.7139 & 0.7260 \\
1.9 & 0.7517 & 0.7508 & 0.7489 & 0.7139 & 0.7260 \\
2.0 & 0.7498 & 0.7485 & 0.7549 & 0.7139 & 0.7260 \\
\hline
\end{tabular}

can be easily generated with least effort. This work can be extended for the three-dimensional analysis of complex fluid flow problems using EFG method.

\section{List of symbols}

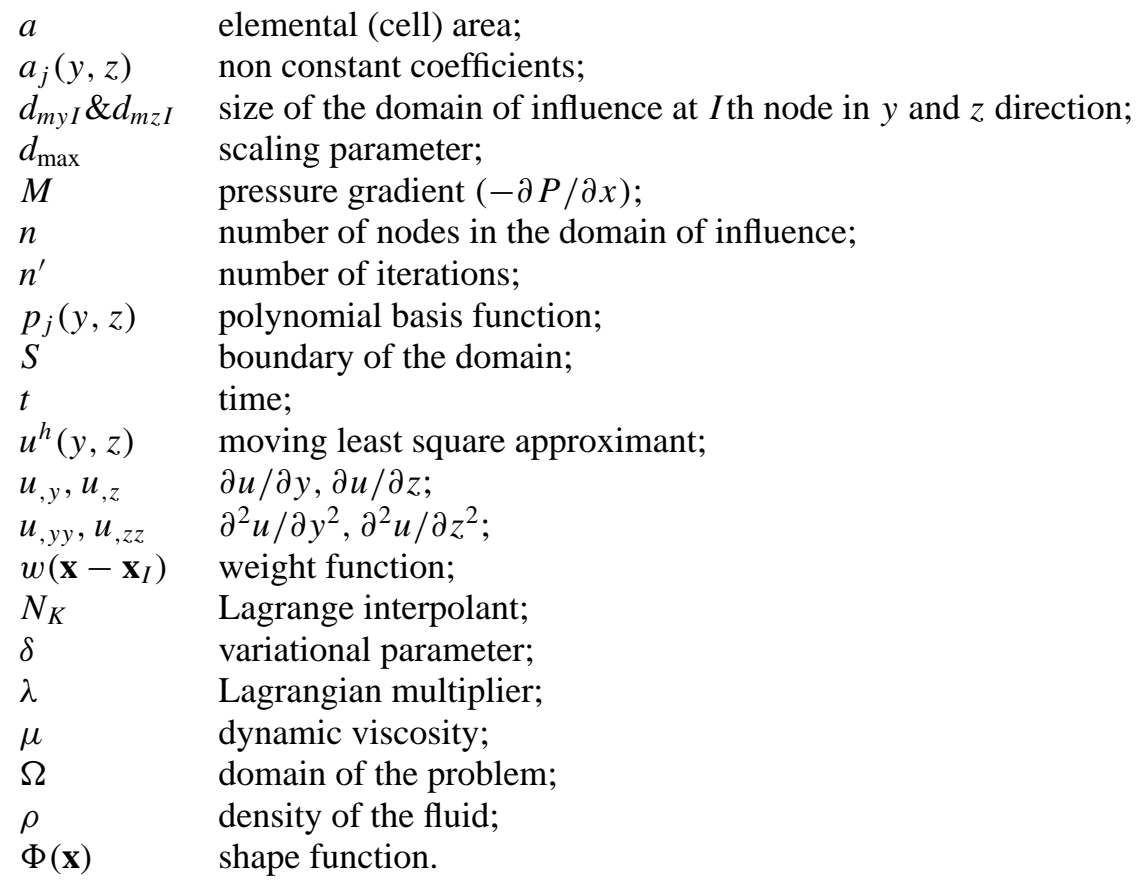




\section{References}

Appel D W, Hubbard P G, Landweber L, Laursen E M, McNown J S, Rouse H, Siao T T, Touch A, Yih C S 1959 Advanced mechanics of fluids (Iowa: Institute of Hydraulic Research)

Atluri S N, Zhu T 1998 A new meshless local Petrov-Galerkin (MLPG) approach in computational mechanics. Comput. Mech. 22: 117-127

Babuska I, Melenk J M 1997 The partition of unity method. Int. J. Numer. Methods Eng. 40: 727-758

Belytschko T, Tabbara M 1996 Dynamic fracture using element-free Galerkin methods. Int. J. Numer. Methods Eng. 39: 923-938

Belytschko T, Lu Y Y, Gu L 1994a Element-free Galerkin methods. Int. J. Numer. Methods Eng. 37: 229-256

Belytschko T, Gu L, Lu Y Y 1994b Fracture and crack growth by element-free Galerkin methods. Model. Simul. Mater. Sci. Eng. 2: 519-534

Belytschko T, Organ D, Krongauz Y 1995 A coupled finite element-element-free Galerkin method. Comput. Mech. 17: 186-195

Belytschko T, Krongauz Y, Organ D, Fleming M, Krysl P 1996 Meshless methods: an overview and recent developments. Comput. Methods Appl. Mech. Eng. 139: 3-47

Cingoski V, Miyamoto N, Yamashita H 1998 Element-free Galerkin method for electromagnetic field computations. IEEE Trans. Magn. 34: 3236-3239

De S, Bathe K J 2000 The method of finite spheres. Comput. Mech. 25: 329-345

Dolbow J, Belytschko T 1998 An introduction to programming the meshless element-free Galerkin method. Arch. Comput. Mech. 5: 207-241

Krysl P, Belytschko T 1996a Analysis of thin plates by element-free Galerkin method. Comput. Mech. 17: $26-35$

Krysl P, Belytschko T 1996b Analysis of thin shells by element-free Galerkin method. Int. J. Solids Struct. 33: 3057-3080

Liu W K, Jun S, Zhang Y F 1995 Reproducing kernel particle methods. Int. J. Numer. Methods Eng. 20: 1081-1106

Liu G R, Gu Y T 2000 Coupling of element-free Galerkin method and hybrid boundary element methods using modified variational formulation. Comput. Mech. 26: 166-173

Lu Y Y, Belytschko T, Gu L 1994 A new implementation of the element-free Galerkin method. Comput. Methods Appl. Mech. Eng. 113: 397-414

Lu Y Y, Belytschko T, Tabbara M 1995 Element-free Galerkin methods for wave propagation and dynamic fracture. Comput. Methods Appl. Mech. Eng. 126: 131-153

Monaghan J J 1988 An introduction to SPH. Comput. Phys. Commun. 48: 89-96

Monaghan J J 1992 Smoothed particle hydrodynamics. Annu. Rev. Astron. Astrophys. 30: 543-574

Nayroles B, Touzot G, Villon P 1992 Generalizing the finite element method: diffuse approximation and diffuse elements. Comput. Mech. 10: 307-318

Reddy J N 1993 An introduction to the finite element method (McGraw-Hill: Singapore)

Singh I V, Sandeep K, Prakash R 2002 The element-free Galerkin method in three-dimensional steady state heat conduction. Int. J. Comput. Eng. Sci. 3: 291-303

Singh I V, Sandeep K, Prakash R 2003a Heat transfer analysis of two-dimensional fins using meshless element-free Galerkin method. Numer. Heat Transfer A44: 73-84

Singh I V, Sandeep K, Prakash R 2003b Meshless EFG method in transient heat conduction problems. Int. J. Heat Technol. 21: 99-105

Sukumar N, Moran B, Black T, Belytschko T 1997 An element-free Galerkin method for threedimensional fracture mechanics. Comput. Mech. 20: 170-175

Sukumar N, Moran B, Belytschko T 1998 The natural element method in solid mechanics. Int. J. Numer. Methods Eng. 43: 839-887

Sukumar N, Moran B, Yu Semenov A, Belikov V V 2001 Natural neighbour Galerkin methods. Int. J. Numer. Methods Eng. 50: 1-27 
Xuan L, Zeng Z, Shanker B, Udpa L 2001 Meshless element-free Galerkin method in NDT applications. 28th Annu. Rev. of Progress in Quantitative and Nondestructive Evaluation, Maine, USA (AIP Conf. Proc.)

Yagawa G, Yamada T 1996 Free mesh method, a new meshless finite element method. Comput. Mech. 18: 383-386

Zhu T, Zhang J D, Atluri S N 1998 A meshless local boundary integral equation (LBIE) method for solving nonlinear problems. Comput. Mech. 22: 174-186 